\title{
MACHINE LEARNING CLUSTERING FOR POINT CLOUDS OPTIMISATION VIA FEATURE ANALYSIS IN CULTURAL HERITAGE
}

\author{
L. M. Gujski ${ }^{\mathrm{a}}$, A. di Filippo ${ }^{\mathrm{a}}$, M. Limongiello ${ }^{\mathrm{a}}$ \\ ${ }^{a}$ DICIV, Department of Civil Engineering, University of Salerno, Fisciano (SA), Italy \\ (lgujski; anddifilippo1; mlimongiello)@unisa.it.
}

\section{Commission II}

KEY WORDS: photogrammetry, accuracy, point cloud, SOM, K-means

\begin{abstract}
:
The paper presents an innovative approach that can assist survey methods by applying AI algorithms to improve the accuracy of point clouds generated from UAV images. Firstly, the work individually analyses several photogrammetric accuracy parameters, including reprojection error, angle of intersection between homologous points, number of cameras for single Tie Point calculation, verifying that a single parameter is not sufficient to filter noise from a photogrammetric point cloud. Therefore, some of the calculated parameters were analysed with the Self-Organizing Map (SOM) and a K-means, to check the impact of the precision parameters for reducing the noise associated with the definition of the $3 \mathrm{D}$ model. In the case study, in both machine learning clustering algorithms used, it was observed that the parameter that most influences noise in photogrammetric point clouds is the angle of intersection.
\end{abstract}

\section{INTRODUCTION}

In the past ten years, the application of photogrammetry in digital $3 \mathrm{D}$ recording has grown greatly. In fact, due to the development of computer vision technology and new computing technology, photogrammetry technology has accelerated the processing time and realized automation, which was once a well-known weakness (Falkingham, 2012).

With the use of automatic Structure of Motion technology (SfM), the overall situation has gradually changed from the widespread use of scientific applications of $3 \mathrm{D}$ measurement using laser scanner technology to the use of photogrammetry. Nowadays, photogrammetry technology has gained greater "robustness", possibly overcoming distance-based sensors in many applications. Recently, the technological development of Unmanned Aerial Vehicles (UAV) has become easier to pilot and more reliable, which indirectly promotes the growth of photogrammetric applications, especially in medium and large applications.

In the literature, UAV systems used for photogrammetric purposes are usually applied for three-dimensional surveys of complex structures in the Cultural Heritage field. Compared with TLS - Terrestrial Laser Scanning (De Blasiis et al., 2020), the main factors in favour of using drones for acquisition are: lower instrument costs, faster field data collection, and first of all, better ratios 3D model necessary for correct analysis of colour results and archaeological identification (Rinaudo et al., 2012; Barba et al., 2019).

Especially in the field of Cultural Heritage $(\mathrm{CH})$, the applications of photogrammetry for different purposes are numerous (Themistocleous et al., 2015). Due to the speed of acquisition and the transportability of the vehicle, the technology is indeed very versatile, allowing these instruments to be used in different applications (Fernández-Hernandez et al., 2015). In the literature, it is possible find applications for structural monitoring of historical buildings (Arias et al., 2005), used to generate 3D models for volume calculations and then to generate metric maps for presentation purposes for mining estimation (Bendea et al., 2007), also used to draw the degradation of the exterior walls of historical buildings (Brunetaud et al., 2012; Themistocleous, 2017).
A well-known problem in photogrammetry projects is indeed the georeferencing stage, especially the choice of instruments and the way they are employed to obtain maximum accuracy on the model. In fact, after knowing the definition of graphical error, the maximum representation ratio is inversely proportional to the metric error calculated on the model. The low accuracy of the model may invalidate the high resolution of the data, thereby invalidating the graphic scale of the product (i.e., plan, section, elevation). It should be emphasized that according to the final purpose of photogrammetry, the accuracy required for data collection and processing is different; if you want to generate 3D models for augmented reality (AR), or perform simple Web visualization (represented in non-scientific applications), the estimation of model accuracy can be omitted. In the $\mathrm{CH}$ domain (the purpose of which is to protect and/or monitor archaeological heritage or architectural restorations), the accuracy of the metric system must be evaluated to avoid "incorrect" documentation from the metric perspective.

The accuracy of the model is subject to certain photogrammetric boundaries. One of the factors that affect the output accuracy the most is the angle formed between the homologous rays in different cameras. Generally, the larger the angle (within a certain interval), the higher the achievable accuracy, Kraus research shows that there is a direct proportional relationship between the Base/Height ratio and accuracy (Kraus, 2011). Evaluating the accuracy of a georeferenced $3 \mathrm{D}$ model can be done in several ways. A basic method is to analyse the residues of the bundle adjustment by computing the Root Mean Squared Error (RMSE) of the residues on the GCPs or by using the coordinates measured on the ground independently of the points with which to compare the coordinates measured on the photogrammetric model (Check Point) (Sanz-Ablanedo et al., 2018).

In order to evaluate the accuracy of the final model, we will propose an outlier detection method that does not consider a single parameter related to accuracy, but simultaneously all calculated parameters, by applying separately two clustering machine learning algorithms - Self Organizing Map (SOM) and $\mathrm{K}$-means, to reach a compromise model between the data of points available and the noise reduction associated with the $3 \mathrm{D}$ definition. 


\section{BACKGROUND AND QUALITY FEATURE}

The evaluation of the quality of the photogrammetric design within SfM methods can be done using several features, derived both in the acquisition phase (i.e., number of images contributing to the $3 \mathrm{D}$ reconstruction of a Tie Point or the angle of intersection of homologous rays) and in the image processing phase (i.e., reprojection error) (Farella et al., 2020).

In this work the analysed features are reported below. First of all, a single parameter analysis was carried out in order to highlight that a filtering approach on a single feature is not able to clean the point cloud from noise.

\subsection{Reprojection Error}

A geometric error corresponding to the image distance between the projected point and the measured point (James et al., 2017) is the reprojection error. It is used to measure how accurately a 3D point estimate recreates the true projection of the point. In order to calculate the 3D coordinates of the Tie Point, the internal and external direction parameters of the camera and the image coordinates of the point are used. Once its coordinates are calculated, the $3 \mathrm{D}$ point will be reprojected on all the images where it appears. The reprojection error is the difference between the image-point and the reprojected point on a single image. This error is also called RMS image residual. Theoretically, reprojection error is obtained as follows:

$$
\begin{array}{r}
\left(\begin{array}{l}
u \\
v \\
1
\end{array}\right)=\left(\begin{array}{ccc}
f_{u} & 0 & u_{c} \\
0 & f_{v} & v_{c} \\
0 & 0 & 1
\end{array}\right)\left(R_{c}^{T}-R_{c}^{T} t_{c}\right)\left(\begin{array}{l}
P \\
1
\end{array}\right)=K P_{c}\left(\begin{array}{c}
P \\
1
\end{array}\right) \\
\left.\varepsilon_{i}=\left(\begin{array}{c}
u_{i} \\
v_{i}
\end{array}\right)-\left[\begin{array}{lll}
0 & 0 & 1
\end{array}\right) K P_{c}\left(\begin{array}{c}
p_{i} \\
1
\end{array}\right)\right]^{-1}\left(\begin{array}{lll}
1 & 0 & 0 \\
0 & 1 & 0
\end{array}\right) K P_{c}\left(\begin{array}{c}
p_{i} \\
1
\end{array}\right)
\end{array}
$$

where $\quad f_{u}, f_{v}=$ focal lengths in the $u$ and $v$ directions $u_{c}, v_{c}=$ principal point offset $t_{c}=$ position of the camera centre in the object space $R_{c}=$ rotation from the camera

$K=$ matrix of internal parameters $P=$ vector projected space coordinate $p_{i}$ $K P_{c}=$ represents the projection matrix

Each extracted Tie Point is assigned a reprojection error value $\varepsilon$ that is the module of the sum of the reprojection errors computed for the number of cameras that observe the i-th Tie Point. The frequency distribution of the reprojection errors that better fit the data was analysed using MATLAB (i.e., the Statistics toolbox). The distribution was used to exclude external values which are considered outliers at a selected experimental threshold. The algorithm implemented in the Python environment has been used to remove the binding point corresponding to the threshold that identifies it as TP.

\subsection{Angle between homologous points}

In this article, by estimating the angle between the two projection lines (called the "intersection angle"), the Base/Height ratio is analysed. The photogrammetry software we use does not give the value of this angle in the output, so we implemented an algorithm in the Python environment.

In order to extract and calculate the parameters of interest, two libraries are used: NumPy (Oliphant, 2007), which is used to add support for large multi-dimensional arrays and matrices, and a large collection of advanced mathematical functions for operations on these arrays; Pandas (McKinney, 2010) is a BSDlicensed open source library that provides high-performance, easy-to-use data structures. Projection Centre $(O)$ and Tie Point $(k)$ are the input parameters used to calculate the angle of intersection. Given the k-th Tie Point seen from two images $i$ and $j$, the direction vectors $u_{i}$ and $v_{j}$ are given by the relations:

$$
\begin{aligned}
& u_{i}=\left[E_{O i}-E_{k} ; N_{O i}-N_{k} ; h_{O i}-h_{k}\right] \\
& v_{j}=\left[E_{O j}-E_{k} ; N_{O j}-N_{k} ; h_{O j}-h_{k}\right]
\end{aligned}
$$

where the subscript $O_{i}$ indicates the projection centre of the i-th frame and $O_{j}$ of the j-th frame, $E, N, h$ are the cartographic coordinates. The relation gives the intersection angle $\alpha$ :

$$
\cos \alpha=\frac{u \cdot v}{|u| \cdot|v|}
$$

The intersection angle calculation was made using all the image pairs that see the i-th Tie Point, calculating the intersection angle for each pair and finally calculating the average intersection angle between the $\mathrm{n}$ frames that see the point, removing the extreme values. Finally, with each Tie Point extracted, the method associates the average angular value obtained. The whole process is implemented in Python.

\subsection{Image redundancy}

This parameter is the number of photogrammetric shots implemented within the SfM process, for the reconstruction of the i-th TP in 3D space. With the same other parameters of photogrammetric accuracy, it is assumed that as the image redundancy increases, the metric quality of the TP point cloud improves.

$$
N_{i}=\sum_{j} n_{j_{T P i}}
$$

where $\quad n_{j_{T P i}}=$ camera for the reconstruction of the i-th TP.

\subsection{Projection Accuracy}

This parameter is the point placement from local neighbour points. The accuracy of Tie Point projections depends on the scale at which they were located. Metashape uses information about scale to weight Tie Point reprojection errors. Key point size is the Sigma of the Gaussian blur at the pyramid level of scales at which the key point was found (Mirko et al., 2019).

\section{CASE STUDY}

The case study considered developing this work is the temple of Neptune (Figure 1), surveyed with aerial photogrammetry by UAV in 2017: the complex spatial articulation of the geometries, make it a suitable stage for the type of qualitative analysis carried out. The photogrammetric survey was carried out exclusively by drone. A mixed type of photogrammetric acquisition was chosen: the nadiral images were acquired through the generation of a flight plan designed in the DJI Ground Station environment, while for the oblique images necessary for the 3D reconstruction of the entire Temple and for the restitution of the orthophotos of the elevations - a manual piloting mode was adopted. 


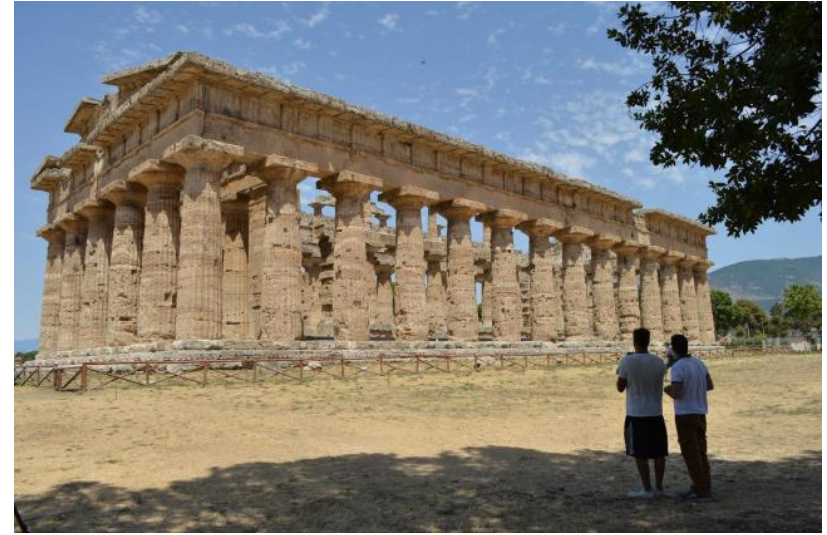

Figure 1. Photogrammetric survey phase.

For both phases, the acquisition was performed in automatic timelapse ( $2 \mathrm{sec}$ interval). The UAV used is a hexacopter assembled with a 3-axis gimbal and the installation of a Sony Alpha 6500 camera (sensor size $23.5 \times 15.6 \mathrm{~mm}, 6000$ x 4000 pixels, pixel size $3.92 \mu \mathrm{m}$, focal length $16 \mathrm{~mm}$ ). During the flight operations, access to the Temple and the area nearby security buffer - was closed to the public for a period of 2 hours. The nadiral flight was from North-East to South-West, with a height from the take-off plane of $35 \mathrm{~m}$, which resulted in a ground cover of $51.4 \times 34.1 \mathrm{~m}$. In total - from the flight plane only - 185 nadiral images were acquired. The manual flight for the oblique images was planned by carrying out two strips for each side of the Temple: the first was carried out with the camera at $45^{\circ}$ - with an average flight altitude of $17.5 \mathrm{~m}$ from the take-off point - so that each photogrammetric shot would take a percentage of the frame of the upper part of the Temple, so as to overlap with the nadiral images, as much as the external part of the columns; the other strip at a lower altitude - an average of $13.5 \mathrm{~m}$ from the take-off plane - has instead planned a horizontal optical axis of the camera, to guarantee an overlap of the photogrammetric shot with the images acquired in the first strip of between 60 and $80 \%$. Finally, a set of images were acquired at a higher altitude - between 45 and $55 \mathrm{~m}$ - to facilitate the matching process in the processing phase. A total of 908 images were processed for the generation of the 3D model (185 nadiral images obtained from the flight plan and 723 oblique images obtained in manual mode).

The images were processed in Agisoft Metashape (version 1.7.1 build 11797). The workflow implemented is as follows: in the Align Photos phase, the parameters were set: Accuracy $=$ High, Key point limit $=60.000$, Tie Point limit $=$ no limit, obtaining $\mathrm{a}$ Sparse Cloud of 2.626.415 points (Figure 2). In the creation of the Dense Cloud the parameters used were: Quality = High, Depth filtering $=$ Disable, returning a Dense Cloud of 56.150.877 points. In the Build Mesh the parameters set are Surface Type $=$ Arbitrary, Source Data $=$ Dense Cloud, Face Count $=$ Medium, generating a polygonal model with 5.230.012 triangles. Finally, in Build Texture the parameters set are: Mapping Mode $=$ Generic, Blending Mode $=$ Mosaic, Texture size $=4096 \times 1$ (enabling Enable hole fitting). A GNSS network consisting of 11 Ground Control Points (GCPs), staggered altimetrically, was designed to support the georeferencing and accuracy assessment of the generated model. The GCPs were materialized on the ground using photogrammetric targets and topographic pegs. The reference system adopted is UTM/ETRF00 with orthometric heights. The precision achieved in planimetry is on average subcentimetric while in altimetry it is about $2.5 \mathrm{~cm}$.

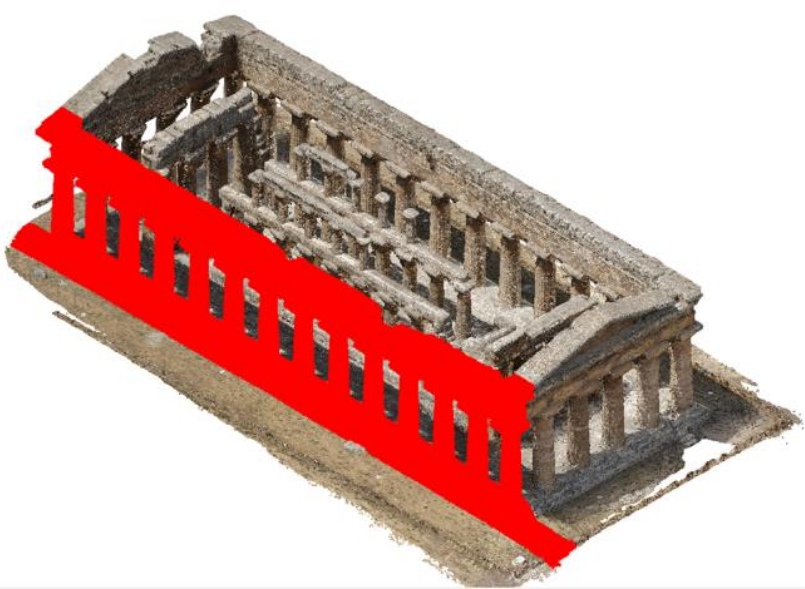

Figure 2. Photogrammetric Point cloud and section analysed (in red).

The GCPs were included for the estimation of the internal orientation parameters in Agisoft Metashape using a selfcalibrating bundle adjustment. These estimated parameters were then used to orient the images and kept constant throughout SfM processing.

\section{MACHINE LEARNING CLUSTER}

Unsupervised learning, also known as unsupervised machine learning (Friedman et al., 2001), analyses and clusters unlabelled information using machine learning techniques. Without the need for human interaction, these algorithms uncover hidden patterns or data groupings.

Unsupervised learning models are utilized for three fundamental tasks: clustering (Madhulatha, 2012; Farella et al., 2019), association, and dimensionality reduction. Clustering is the most significant unsupervised learning problem, and it deals with discovering a structure in a collection of unlabelled data.

\subsection{SOM}

As an artificial neural network, the self-organizing map (SOM) (Kohonen, 2001) is commonly used for display and analysis of high-dimensional data.

SOM is utilized not just for visualization, but also for data clustering. As multi-dimensional scaling, SOM may be integrated with dimensionality reduction approaches (Kurasova and Molytè, 2011).

In the iterative algorithm each node's weights are initialized. At random, a vector is selected from the training data set. To determine which weights are most similar to the input vector, every node is examined.

The winning node is generally referred to as the Best Matching Unit (BMU). The BMU neighbourhood is then calculated. The number of neighbours reduces over time. The winning weight is rewarded by being more like the vector in the sample.

The neighbours become more like the vector of the sample, too. The closest a node is to the BMU, the less it knows, the more its weights are altered and the farther distant the neighbour is from the BMU. 


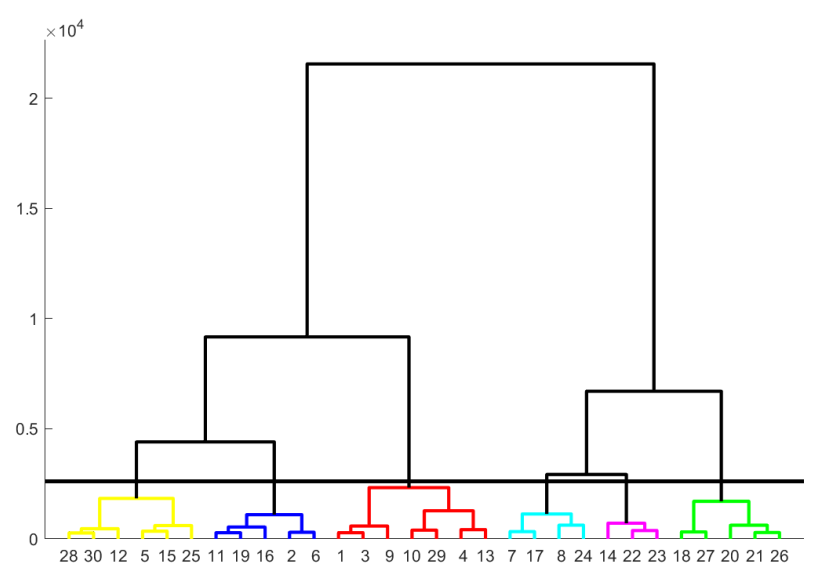

Figure 3. Dendrogram, clusters for the data $=6$.

The whole process is implemented in MATLAB, using the Neural Network Clustering App. The choice of the number of clusters to be brought into the accuracy analysis is made by the graphical representation of the dendrogram (Figure 3). A dendrogram is a diagram representing a tree that shows the hierarchical relationship between object and used to visualise the similarity in the clustering process. In clustering techniques, the dendrogram is used to provide a graphic representation.

\subsection{K-means}

$\mathrm{K}$-means is a technique that divides a dataset into a set of groups based on the number of clusters supplied by the user (Lloyd, 1982). The program examines the data to identify data points that are organically similar and assigns each point to a cluster of points with similar features. The data can then be labelled into different classes based on the features of each cluster. The algorithm converges when there is no further change in assignment of instances to clusters. The whole process is implemented using Scikit-learn (Pedregosa et al., 2011), a free machine learning library for Python.

Finding the optimal number of clusters is an important part of this algorithm. A commonly used method for finding optimal $\mathrm{K}$ value is Elbow Method (Bholowalia and Kumar, 2014).

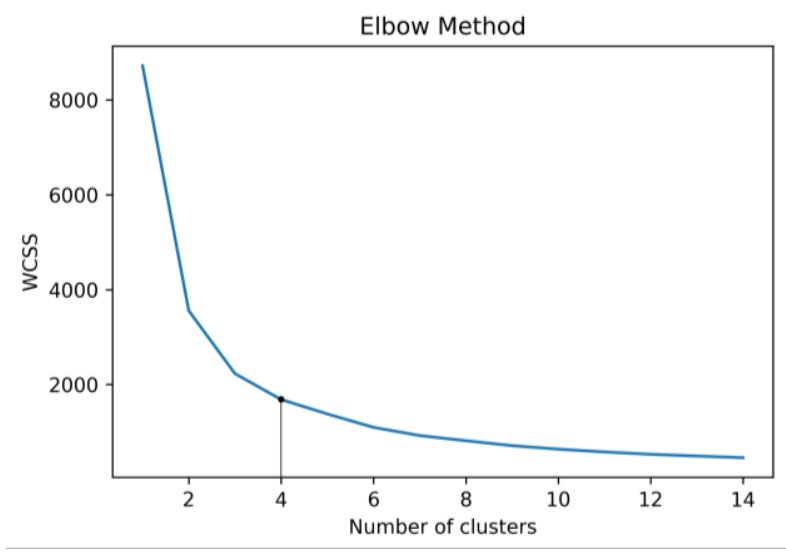

Figure 4. Elbow method, clusters for the data $=4$.

In the Elbow method (Figure 4), the idea is to vary the number of clusters (K) from 1 to 15 for example. The WCSS (WithinCluster Sum of Square) is calculated for each $\mathrm{K}$ value. The sum of squared distances between each point in a cluster and its centroid is WCSS.

$$
W C S S=\sum_{C_{k}}^{C_{n}} \sum_{d_{i} i n C_{i}}^{d_{m}} \operatorname{distance}\left(d_{i}, C_{k}\right)^{2}
$$

where $\quad C=$ cluster centroids

$d=$ data point in each cluster

The WCSS value will begin to decrease as the number of clusters increase. When examining the graph, we can see that it shifts rapidly at one point, forming an elbow. At this point, the graph begins to move almost parallel to the $\mathrm{X}$-axis. The optimal value of $\mathrm{K}$, or the optimal number of clusters, corresponds to this point. Thus, for our data, we conclude that the optimal number of clusters for the data is 4 .

\section{RESULT FEATURE ANALYSIS}

In the first step we considered the parameters individually to see how the filtering procedures influenced the accuracy value. The first parameter considered is the error projection. For the purposes of the analysis, a standard section was identified, 2.5 $m$ wide, visible in the Figure 2 in red.

Using a MATLAB tool (i.e., the Statistics toolbox), the bestfitting frequency distribution of the reprojection errors was studied. The best-fitting distribution was used to remove values outside a chosen experimental threshold (90, 95 and 99 percentile), in which outliers were considered contained. Tie Points belonging to this threshold are considered as Tie Point outliers and removed automatically with an algorithm implemented in Python. Observing Figure 5 it is possible to notice that filtering the point cloud by analysing only the error projection with a statistical approach does not generate a good degree of filtering for the section under examination; in fact, the procedure removes some mostly isolated points, but does not lead to great advantages in noise reduction. However, most of the isolated points have not been filtered out and, in addition, $3 \mathrm{D}$ points that actually belong to the section are also removed.
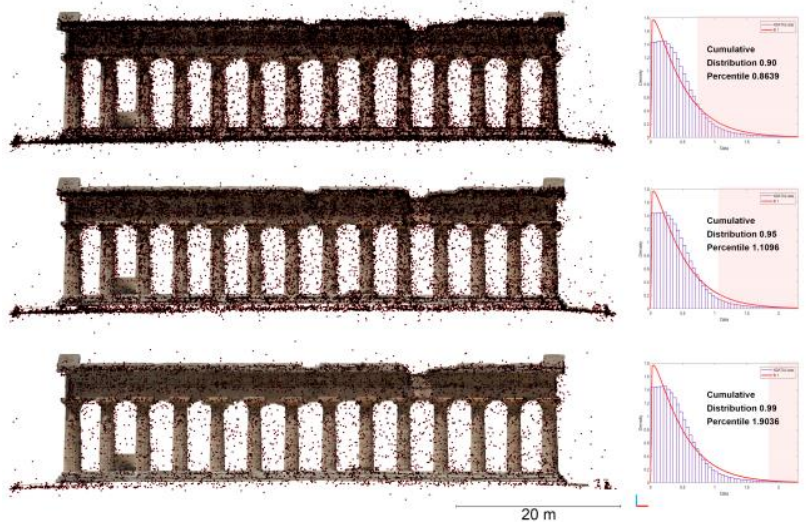

Figure 5. Filtering of reprojection error parameter on values above the lower cumulative distribution.

A better result for noise reducing is obtained by filtering the point cloud according to the average intersection angle, calculated for each Tie Point, and then analysing the acquisition geometry. Excluding small average intersection angles resulted in much more realistic and less noisy surfaces.

In addition, pushing the filter too far by setting average angles as a threshold compromises the amount of data necessary for representation; in fact, setting an average angle of over $18^{\circ}$ as a threshold removes large quantities of points belonging to the columns, making the object of the survey no more distinguishable. 

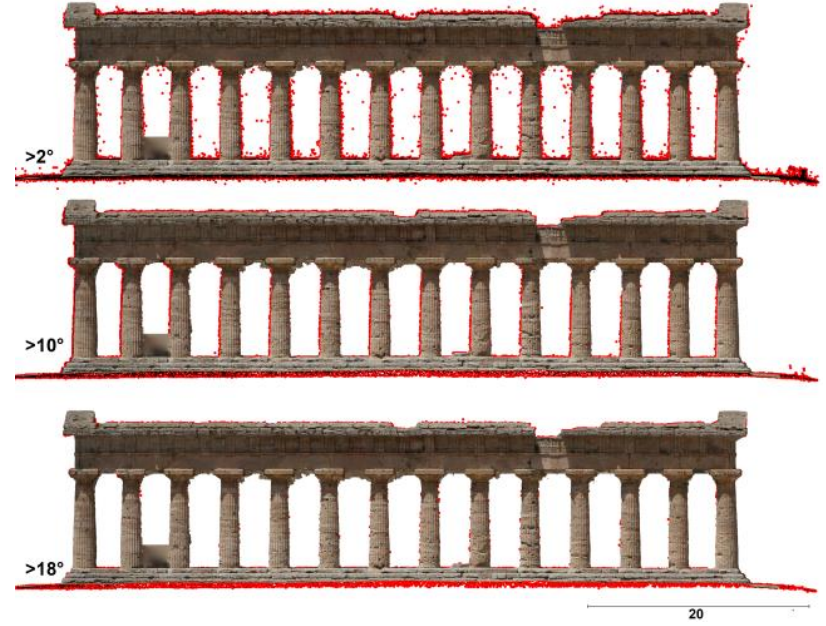

Figure 6. Filtering of the average angle between homologous points for assigned angular values.

For the case study in question, a good reduction in noise on Tie Points is already evident for angles greater than $10^{\circ}$ (Figure 6). Another parameter analysed individually is the number of images, i.e., the quantity of images that have contributed to the reconstruction of the $3 \mathrm{D}$ point in the space. This value is correlated to the number of images in which a point has been measured, therefore, at least in theory it is assumed that the greater the number of images, the better the quality of the 3D point - always supposing that the angle of intersection is satisfactory. From Figure 7 we observe that for a minimum number of images in the range [2-4] the amount of noise included is high, while for a range of [5-9] cameras, the noise of the point cloud tends to decrease. Also, in this case for values greater than the 10 cameras for the reconstruction of the 3D point, the quantity of points in the section is drastically reduced, eliminating not only the noise but also portions of columns, damaging the quantity of the data.

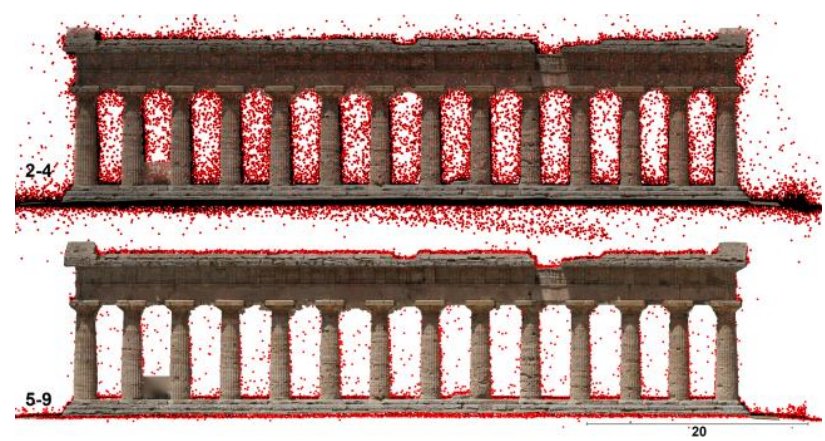

Figure 7. Image count filter: on top for values between [2-4], on the bottom for values between [5-9].

Finally, the last parameter analysed individually is the projection accuracy. The trend of this parameter's filtering is similar to that of the image count: as the filtering threshold value increases, the noise decreases, but increasing this value above a limit eliminates portions of the columns, damaging the quality of the data.

It can be seen in Figure 8 that the limit parameter for noise reduction is an accuracy reprojection equal to 10: increasing this value further reduces the noise threshold but at the same time also reduces portions of the edited cloud.

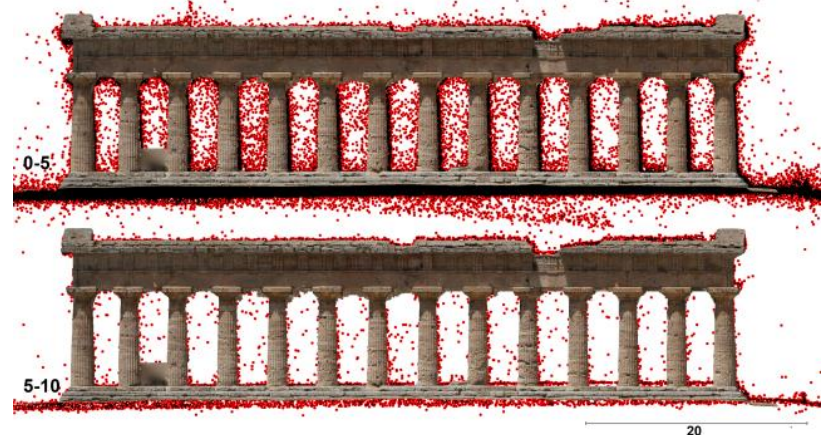

Figure 8. Projection Accuracy filter: on top for values between [0-5], bottom for values between [5-10].

\section{RESULT CLUSTER ANALYSIS}

The clustering algorithms were chosen to consider not only one accuracy variable but all measurable parameters at the same time. The reprojection error, projection accuracy, number of pictures, and average angle were utilized as input data. The choice of cluster membership in the High accuracy or Noise group was made by checking the average angle values (from what was observed in the single feature analysis, the angle value is the one that most affects noise). Clusters with larger angles were placed in High accuracy, averaged over the number of Tie Points belonging to the cluster, and the group called "Noise", which includes the clusters with smaller average angular values. It can be noted that the High layer turns out to be the best fitting point set for the both cases SOM (Figure 9) and K-means (Figure 10).

\subsection{SOM}

The SOM Layer loaded with an $8 \times 8$ network and 100 epochs. The graphical dendrogram (Figure 3 ) is used to determine the number of clusters for accuracy analysis. Six categories can be recognized when the dendrogram is generated. The average values of the various analysed features belonging to the High accuracy and Noise groups are shown in Table 1.

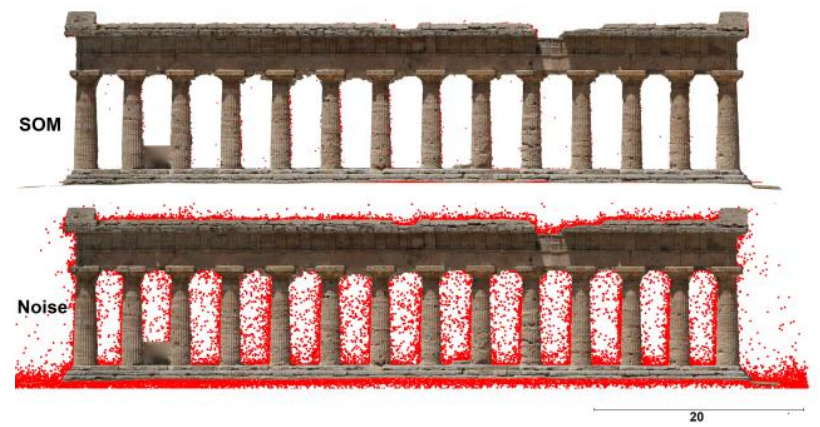

Figure 9. Cluster analysis and filtering by SOM: on top high accuracy cluster, on bottom for noise cluster.

\subsection{K-means}

With the Scikit-learn library all the data was clustered by KMeans clustering algorithm. using four as the number of clusters obtained after analysis of the elbow graph (Figure 4), 10 the number of time that the k-means algorithm will be run with different centroid seeds, and 300 as the maximum number of iterations of the k-means algorithm for a single run. The average values of the various analysed features belonging to the High accuracy and Noise groups are shown in Table 2. 


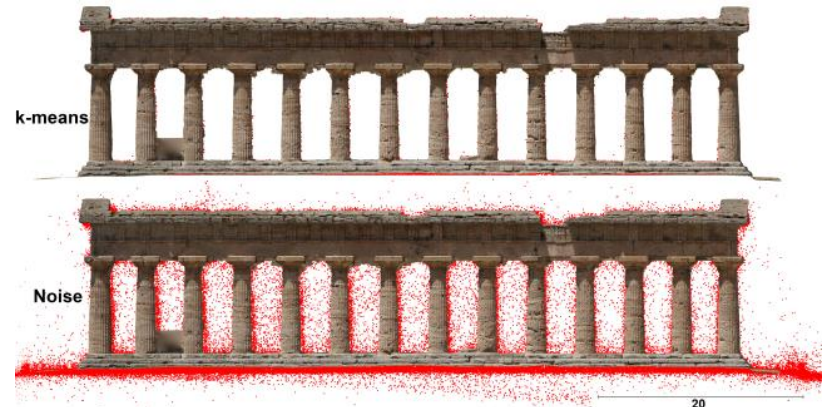

Figure 10. Cluster analysis and filtering by k-means: on top high accuracy cluster, on bottom for noise cluster.

\begin{tabular}{|c|c|c|c|c|c|c|}
\hline \multirow{15}{*}{ 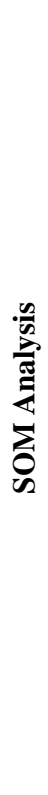 } & & E_Proj & Acc_Pr & N_Im & Angle $^{\circ}$ & \\
\hline & Mean & 0,59 & 6,55 & 6 & 18,44 & \multirow{7}{*}{ 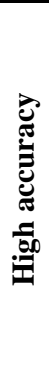 } \\
\hline & std & 0,69 & 51,13 & 8 & 6,73 & \\
\hline & min_value & 0,00 & 0,00 & 2 & 1,14 & \\
\hline & $25 \%$ perc & 0,29 & 0,29 & 3 & 14,01 & \\
\hline & $50 \%$ perc & 0,48 & 1,06 & 4 & 16,97 & \\
\hline & $75 \%$ perc & 0,71 & 3,09 & 6 & 21,26 & \\
\hline & max_value & 28,69 & 5209,53 & 184 & 115,91 & \\
\hline & Mean & 0,38 & 1,40 & 3 & 3,44 & \multirow{7}{*}{$\frac{\mathscr{n}}{\frac{0}{0}}$} \\
\hline & std & 0,44 & 12,25 & 2 & 1,55 & \\
\hline & min_value & 0,00 & 0,00 & 2 & 0,05 & \\
\hline & $25 \%$ perc & 0,14 & 0,04 & 2 & 2,37 & \\
\hline & $50 \%$ perc & 0,29 & 0,21 & 2 & 3,56 & \\
\hline & $75 \%$ perc & 0,50 & 0,83 & 3 & 4,56 & \\
\hline & max_value & 15,91 & 2217,85 & 51 & 15,47 & \\
\hline
\end{tabular}

Table 1. SOM clustering: feature parameter analysis.

\begin{tabular}{|c|c|c|c|c|c|c|}
\hline \multirow{15}{*}{ 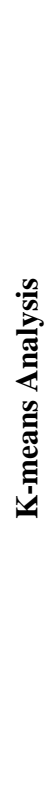 } & & E_Proj & Acc_Pr & N_Im & Angle $^{\circ}$ & \\
\hline & Mean & 0,62 & 7,81 & 8 & 29,85 & \multirow{7}{*}{ 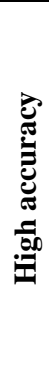 } \\
\hline & std & 0,58 & 55,33 & 10 & 14,68 & \\
\hline & min_value & 0 & 0 & 2 & 1,99 & \\
\hline & $25 \%$ perc & 0,33 & 0,32 & 2 & 18,36 & \\
\hline & $50 \%$ perc & 0,53 & 1,33 & 4 & 27,27 & \\
\hline & $75 \%$ perc & 0,76 & 4,41 & 8 & 36,96 & \\
\hline & max_value & 30,14 & 6418,10 & 129 & 131,79 & \\
\hline & Mean & 0,40 & 1,46 & 3 & 2,22 & \multirow{7}{*}{$\frac{n}{0}$} \\
\hline & std & 0,48 & 10,96 & 2 & 1,43 & \\
\hline & min_value & 0 & 0 & 2 & 0,05 & \\
\hline & $25 \%$ perc & 0,14 & 0,04 & 2 & 0,96 & \\
\hline & $50 \%$ perc & 0,30 & 0,23 & 2 & 1,99 & \\
\hline & $75 \%$ perc & 0,51 & 0,86 & 3 & 3,39 & \\
\hline & max_value & 20,03 & 1725 & 62 & 5,19 & \\
\hline
\end{tabular}

Table 2. K-means clustering: feature parameter analysis.

\section{CONCLUSION}

This work shows that the use of a single parameter in the reduction of noise in a Tie Point cloud is not the best approach. In fact, all the parameters analysed - error projection, angle between homologous points, image count and accuracy projection - show that the filtering process only partially eliminates the noise, or rather the outliers that do not belong to the edited cloud. The parameter that best eliminates outliers without affecting the edited cloud is the angle between homologous points: the problem with this parameter is that as the angle value increases, more outliers are removed, but after a threshold value, points belonging to the edited cloud are simultaneously eliminated so that the density of the cloud does not allow a good description of the object. This parameter depends directly on the image acquisition phase, so this value can vary considerably: in the case study presented, a value above $10^{\circ}$ eliminates most of the outliers, without compromising the density of the edited cloud.

The analysis of the various features by SOM and K-means made it possible to consider - in the process of filtering the cloud - all the parameters analysed individually. In particular, in Table 1 and Table 2, the mean values and standard deviations obtained in the clustering process categorised as High accuracy and Noise class.

With the analysis of -SOM and K-means- the average values between the classes of accuracy and noise are not very far from each other for the features of Error projection, Image count and Accuracy projection, while they vary a lot in the values of angular average for the intersection angles. Moreover, analysing the standard deviation, the class High accuracy has very high values of the features Image count, Accuracy projection (both always only positive) and angular value, while for error projection also the value of standard deviation between the classes of accuracy and noise are very similar.

Finally, it is analysed that the clouds produced by the SOM and K-means analysis for the high accuracy class do not have significant deviations in a cloud-to-cloud comparison, even though the accuracy class produced by K-means has higher average angular values; conversely, from a density point of view, the SOM analysis produces an average cloud twice as dense as that of K-means.

\section{REFERENCES}

Arias, P., Herraez, J., Lorenzo, H., Ordonez, C., 2005. Control of structural problems in cultural heritage monuments using close-range photogrammetry and computer methods. Computers \& structures $83,1754-1766$.

Barba, S., Barbarella, M., Di Benedetto, A., Fiani, M., Limongiello, M., 2019. Comparison of Uavs Performance for a Roman Amphitheatre Survey: The Case of Avella (Italy). 2nd International Conference of Geomatics and Restoration (Geores 2019) 42-2, 179-186.

Bendea, H., Chiabrando, F., Tonolo, F.G., Marenchino, D., 2007. Mapping of archaeological areas using a low-cost UAV. The Augusta Bagiennorum test site, XXI International CIPA Symposium. Citeseer.

Bholowalia, P., Kumar, A., 2014. EBK-means: A clustering technique based on elbow method and k-means in WSN. International Journal of Computer Applications 105.

Brunetaud, X., Luca, L.D., Janvier-Badosa, S., Beck, K., AlMukhtar, M., 2012. Application of digital techniques in monument preservation. European Journal of Environmental and Civil Engineering 16, 543-556. 
De Blasiis, M.R., Di Benedetto, A., Fiani, M., 2020. Mobile Laser Scanning Data for the Evaluation of Pavement Surface Distress. Remote Sensing 12, 942.

Falkingham, P.L., 2012. Acquisition of high resolution threedimensional models using free, open-source, photogrammetric software. Palaeontologia Electronica 15.

Farella, E.M., Torresani, A., Remondino, F., 2019. Sparse point cloud filtering based on covariance features, 27th CIPA International Symposium "Documenting the past for a better future", pp. 465-472.

Farella, E.M., Torresani, A., Remondino, F., 2020. Refining the Joint 3D Processing of Terrestrial and UAV Images Using Quality Measures. Remote Sensing 12, 2873.

Fernández-Hernandez, J., González-Aguilera, D., Rodríguez-Gonzálvez, P., Mancera-Taboada, J., 2015. Image-based modelling from unmanned aerial vehicle (UAV) photogrammetry: an effective, low-cost tool for archaeological applications. Archaeometry 57, 128-145.

Friedman, J., Hastie, T., Tibshirani, R., 2001. The elements of statistical learning. Springer series in statistics New York.

James, M.R., Robson, S., d'Oleire-Oltmanns, S., Niethammer, U., 2017. Optimising UAV topographic surveys processed with structure-from-motion: Ground control quality, quantity and bundle adjustment. Geomorphology 280, 51-66.

Kohonen, T., 2001. The basic SOM, Self-organizing maps. Springer, pp. 105-176.

Kraus, K., 2011. Photogrammetry: geometry from images and laser scans. Walter de Gruyter.

Kurasova, O., Molyte, A., 2011. Quality of quantization and visualization of vectors obtained by neural gas and selforganizing map. Informatica 22, 115-134.

Lloyd, S., 1982. Least squares quantization in PCM. IEEE transactions on information theory 28, 129-137.

Madhulatha, T.S., 2012. An overview on clustering methods. arXiv preprint arXiv:1205.1117.

McKinney, W., 2010. Data structures for statistical computing in python, Proceedings of the 9th Python in Science Conference. Austin, TX, pp. 51-56.

Mirko, S., Eufemia, T., Alessandro, R., Giuseppe, F., Umberto, F., 2019. Assessing the Impact of the Number of GCPS on the Accuracy of Photogrammetric Mapping from UAV Imagery. Baltic Surveying, 43.

Oliphant, T.E., 2007. Python for scientific computing. Computing in science \& engineering 9, 10-20.

Pedregosa, F., Varoquaux, G., Gramfort, A., Michel, V., Thirion, B., Grisel, O., Blondel, M., Prettenhofer, P., Weiss, R., Dubourg, V., 2011. Scikit-learn: Machine learning in Python. the Journal of machine Learning research 12, 2825-2830.

Rinaudo, F., Chiabrando, F., Lingua, A., Spanò, A., 2012. Archaeological site monitoring: UAV photogrammetry can be an answer. International Archives of the Photogrammetry, Remote Sensing and Spatial Information Sciences 39, 583-588.

Sanz-Ablanedo, E., Chandler, J.H., Rodríguez-Pérez, J.R., Ordóñez, C., 2018. Accuracy of unmanned aerial vehicle (UAV) and SfM photogrammetry survey as a function of the number and location of ground control points used. Remote Sensing 10, 1606.
Themistocleous, K., 2017. Model reconstruction for 3d vizualization of cultural heritage sites using open data from social media: The case study of Soli, Cyprus. Journal of Archaeological Science: Reports 14, 774-781.

Themistocleous, K., Ioannides, M., Agapiou, A., Hadjimitsis, D.G., 2015. The methodology of documenting cultural heritage sites using photogrammetry, UAV, and 3D printing techniques: the case study of Asinou Church in Cyprus, Third International Conference on Remote Sensing and Geoinformation of the Environment (RSCy2015). International Society for Optics and Photonics, p. 953510. 